\title{
Shi-type estimates and finite time singularities of flows of $\mathrm{G}_{2}$ structures
}

\author{
Gao Chen
}

April 10, 2018

\begin{abstract}
In this paper, we extend Lotay-Wei's Shi-type estimate from Laplacian flow to more general flows of $\mathrm{G}_{2}$ structures including the modified Laplacian co-flow. Then we prove a version of $\kappa$-non-collapsing theorem. We will use both of them to study finite time singularities of general flows of $\mathrm{G}_{2}$ structures.
\end{abstract}

\section{Introduction}

Let $M$ be a compact 7-manifold. A $\mathrm{G}_{2}$ structure on $M$ is defined by a 3 -form $\phi$ such that at each point there exists an element in $G L(7, \mathbb{R})$ which maps $\phi$ into

$$
e^{123}+e^{145}+e^{167}+e^{246}-e^{257}-e^{347}-e^{356}
$$

where $e^{i j k}=e^{i} \wedge e^{j} \wedge e^{k}$ and $\left\{e^{i}\right\}$ are the standard basis of $T^{*} M$. It induces a metric $g$ by

$$
\left.\left.g(u, v) \operatorname{Vol}_{g}=\frac{1}{6}(u\lrcorner \phi\right) \wedge(v\lrcorner \phi\right) \wedge \phi .
$$

If $\phi$ is closed, it is called a closed $\mathrm{G}_{2}$ structure. If $\psi=* \phi$ is closed, it is called a co-closed $\mathrm{G}_{2}$ structure. For a $\mathrm{G}_{2}$ structure, the torsion tensor $\mathbf{T}$ is defined by

$$
\nabla_{a} \phi_{b c d}=\mathbf{T}_{a}{ }^{e} \psi_{\text {ebcd }}
$$

If the torsion tensor $\mathbf{T}$ vanishes, then it is called a torsion-free $\mathrm{G}_{2}$ structure. The holonomy group of the metric induced by a $G_{2}$ structure is contained in $\mathrm{G}_{2}$ if and only if it is torsion-free.

In order to get general existence results for the torsion-free $\mathrm{G}_{2}$ structures, many versions of flows have been introduced. For example, Bryant 1. proposed the Laplacian flow of closed $G_{2}$ structures:

$$
\frac{\partial}{\partial t} \phi=\Delta_{\phi} \phi
$$

As an analogy, Karigiannis, McKay and Tsui [7 proposed the Laplacian co-flow:

$$
\frac{\partial}{\partial t} \psi=\Delta_{\psi} \psi
$$


However, it is not parabolic. So Grigorian [5] proposed a modified version:

$$
\frac{\partial}{\partial t} \psi=\Delta_{\psi} \psi+2 \mathrm{~d}((A-\operatorname{Tr} \mathbf{T}) \phi),
$$

where $A$ is a suitable constant.

There may be other important flows of $\mathrm{G}_{2}$ structures. In general, they should satisfy the equation

$$
\frac{\partial}{\partial t} \phi_{i j k}=\frac{1}{2} h_{i}^{l} \phi_{l j k} \mathrm{~d} x^{i} \wedge \mathrm{d} x^{j} \wedge \mathrm{d} x^{k}+\frac{1}{6} X^{l} \psi_{l i j k} \mathrm{~d} x^{i} \wedge \mathrm{d} x^{j} \wedge \mathrm{d} x^{k},
$$

where $X$ is a vector field and $h$ is a symmetric tensor. According to Karigiannis [6], the equivalent equation for $\psi$ is

$$
\begin{array}{r}
\frac{\partial}{\partial t} \psi_{i j k l}=h_{i}^{m} \psi_{m j k l}+h_{j}^{m} \psi_{i m k l}+h_{k}^{m} \psi_{i j m l}+h_{l}^{m} \psi_{i j k m} \\
-X_{i} \phi_{j k l}+X_{j} \phi_{i k l}-X_{k} \phi_{i j l}+X_{l} \phi_{i j k} .
\end{array}
$$

The induced equations for the metric and torsion tensor are 6

$$
\frac{\partial}{\partial t} g_{i j}=2 h_{i j}
$$

and

$$
\frac{\partial}{\partial t} \mathbf{T}_{i j}=\mathbf{T}_{i j} h_{m}^{j}+\mathbf{T}_{i j} X^{l} \phi_{l}{ }^{j}{ }_{m}+\left(\nabla^{k} h_{i}^{l}\right) \phi_{k l j}+\nabla_{i} X_{j} .
$$

In this paper, in order to make sure the general flows make sense, we require that

$$
\begin{aligned}
& \frac{\partial}{\partial t} g_{i j}=2 h_{i j}=-2 R_{i j}+C+L(\mathbf{T})+\mathbf{T} * \mathbf{T}, \\
& X=C+L(\mathbf{T})+L(\mathrm{Rm})+L(\nabla \mathbf{T})+\mathbf{T} * \mathbf{T},
\end{aligned}
$$

and

$$
\begin{array}{r}
\frac{\partial}{\partial t} \mathbf{T}_{i j}=\Delta \mathbf{T}_{i j}+L(\mathbf{T})+L(\nabla \mathbf{T})+\mathrm{Rm} * \mathbf{T} \\
+\nabla \mathbf{T} * \mathbf{T}+\mathbf{T} * \mathbf{T}+\mathbf{T} * \mathbf{T} * \mathbf{T},
\end{array}
$$

where $L$ denote linear maps and * denote multi-linear maps. Note that in this paper, we view $\phi, \psi, g$ as constants. For example, $\mathbf{T}^{k l} \psi_{i j k l}$ is considered as $L(\mathbf{T})$. Therefore, we have formulas like

$$
\nabla(L(\mathbf{T}))=L(\nabla \mathbf{T})+\mathbf{T} * \mathbf{T} .
$$

Definition 1.1. In this paper, we call a flow of $\mathrm{G}_{2}$ structures reasonable if it satisfies equations (7), (11), (12), (13), the short time existence and the uniqueness.

For example, for Laplacian flow [9], $X=0$, and

$$
\frac{\partial}{\partial t} g_{i j}=-2 R_{i j}-\frac{2}{3}|\mathbf{T}|^{2} g_{i j}-4 \mathbf{T}_{i}{ }^{k} \mathbf{T}_{k j}
$$

The condition for the torsion is also satisfied. 
For the modified Laplacian co-flow [5], $X=\nabla \operatorname{Tr} \mathbf{T}$, and

$$
\frac{\partial}{\partial t} g_{i j}=-2 R_{i j}+\mathbf{T}^{k m} \mathbf{T}^{l n} \phi_{i k l} \phi_{j m n}+(4 A-2 \operatorname{Tr} \mathbf{T}) \mathbf{T}_{i j}
$$

The condition for the torsion is also satisfied.

The short time existence and uniqueness of the Laplacian flow were proved by Bryant-Xu 2 . The analogous results for the modified Laplacian co-flow were proved by Grigorian [5].

In the case of Laplacian flow, Lotay and Wei 9 proved a global version of Shi-type estimate with respect to $\left(|\mathrm{Rm}(p, t)|_{g(t)}^{2}+|\nabla \mathbf{T}(p, t)|_{g(t)}^{2}\right)^{\frac{1}{2}}$. It is equivalent to $\left(|\operatorname{Rm}(p, t)|_{g(t)}^{2}+|\mathbf{T}(p, t)|_{g(t)}^{4}+|\nabla \mathbf{T}(p, t)|_{g(t)}^{2}\right)^{\frac{1}{2}}$ in that case. The first goal of this paper is to show a local version of Shi-type estimate with respect to $\left(|\mathrm{Rm}(p, t)|_{g(t)}^{2}+|\mathbf{T}(p, t)|_{g(t)}^{4}+|\nabla \mathbf{T}(p, t)|_{g(t)}^{2}\right)^{\frac{1}{2}}$ for all reasonable flows of $\mathrm{G}_{2}$ structures including both the Laplacian flow and the modified Laplacian co-flow. Using the global Shi-type estimate, Lotay-Wei proved that

$$
\sup _{p \in M}\left(|\operatorname{Rm}(p, t)|_{g(t)}^{2}+|\mathbf{T}(p, t)|_{g(t)}^{4}+|\nabla \mathbf{T}(p, t)|_{g(t)}^{2}\right)^{\frac{1}{2}} \geq \frac{C}{T-t},
$$

if $T$ is the maximal existence time for the Laplacian flow. For a reasonable flow of $\mathrm{G}_{2}$ structures, using our Shi-type estimate, (17) is also true.

One may ask whether there are any estimates for the Ricci curvature, scalar curvature and torsion torsion at maximal existence time. The answer is yes. Using the Shi-type estimate and the method of Lotay and Wei, it is easy to see that

$$
\int_{0}^{T} \sup _{M}\left(|\operatorname{Ric}|+|\mathbf{T}|^{2}\right) \mathrm{d} t=\infty
$$

In order to get better estimates using the method of Wang in [12, we need a $\kappa$-non-collapsing theorem. We will show that the $\kappa$-non-collapsing theorem is true if

$$
\int_{0}^{T}(T-t) \sup _{M}|\mathbf{T}|^{4} \mathrm{~d} t<\infty .
$$

In that case, we will prove that

$$
\limsup _{t \rightarrow T}\left[(T-t) \sup _{M}\left(|\operatorname{Ric}|+|\mathbf{T}|^{2}\right)\right]>0,
$$

and

$$
\limsup _{t_{0} \rightarrow T}\left[\left(T-t_{0}\right)^{2} \sup _{t \leq t_{0}}\left(1+|R|+|\mathbf{T}|^{2}\right) \sup _{t \leq t_{0}}\left(|\mathrm{Rm}|+|\mathbf{T}|^{2}+|\nabla \mathbf{T}|\right)\right]>0 .
$$

In particular, if in addition,

$$
\sup _{M}\left(|R|+|\mathbf{T}|^{2}\right)=o\left(\frac{1}{T-t}\right),
$$

then the singularity can not be type-I. In other words,

$$
\sup _{M}\left(|\mathrm{Rm}|+|\mathbf{T}|^{2}+|\nabla \mathbf{T}|\right)=O\left(\frac{1}{(T-t)}\right)
$$


can not be true. Moreover, using our $\kappa$-non-collapsing theorem, we can also show that any blow-up limit near finite-time singularity must be a manifold with holonomy contained in $\mathrm{G}_{2}$ and has maximal volume growth rate.

In Section 2, we prove the Shi-type estimate. In Section 3, we derive the evolution equation for Perelman's $\mathcal{W}$-functional. In Section 4 , we prove the $\kappa$-non-collapsing theorem. In Section 5 we discuss the finite time singularity.

\section{Shi-type estimate}

Theorem 2.1. Let $B_{r}(p)$ be the ball of radius $r$ with respect to $g(0)$ for a reasonable flow of $G_{2}$ structures. Assume the coefficients in the equations (7), (11), (12), (13) are bounded by $\Lambda$. For example, in the modified Laplacian co-flow case, we assume $|A| \leq \Lambda$. If

$$
|\mathrm{Rm}|+|\mathbf{T}|^{2}+|\nabla \mathbf{T}|<\Lambda
$$

on $B_{r}(p) \times[0, T]$, then

$$
\left|\nabla^{k} R m\right|+\left|\nabla^{k+1} \mathbf{T}\right|<C(k, r, \Lambda, T)
$$

on $B_{r / 2}(p) \times[T / 2, T]$ for all $k=1,2,3, \ldots$

Proof. We will use the method proposed by Shi in [11. We start from the evolution equations for the Riemannian curvature, the torsion tensor and their higher order derivatives. It is well known [3] that if $\frac{\partial}{\partial t} g_{i j}=2 h_{i j}$, then

$$
\begin{array}{r}
\frac{\partial}{\partial t} R_{i j k}{ }^{l}=\nabla_{i} \nabla_{k} h_{j p}+\nabla_{j} \nabla_{p} h_{i k}-\nabla_{i} \nabla_{p} h_{j k}-\nabla_{j} \nabla_{k} h_{i p} \\
-R_{i j k}{ }^{q} h_{q p}-R_{i j p}{ }^{q} h_{k p},
\end{array}
$$

and

$$
\frac{\partial}{\partial t} R=-2 \Delta \operatorname{Tr} h+2 \operatorname{div}(\operatorname{div} h)-2<h, \operatorname{Ric}>.
$$

Therefore, let the degree of $\mathbf{T}$ and $\nabla$ be 1 and the degree of $\mathrm{Rm}$ be 2 , then the degree of $\left(\frac{\partial}{\partial t}-\Delta\right) \mathrm{Rm}$ is 4 but it contains no $\nabla^{2} \mathrm{Rm}$ or $\nabla^{3} \mathbf{T}$ term. The degree of $\left(\frac{\partial}{\partial t}-\Delta\right) \mathbf{T}$ is 3 but it contains no $\nabla \mathrm{Rm}$ or $\nabla^{2} \mathbf{T}$ term. The term $\frac{\partial}{\partial t} R-\Delta R-2 \mid$ Ric $\left.\right|^{2}$ is a degree 4 polynomial of Ric, $\nabla^{2} \mathbf{T}, \nabla \mathbf{T}$ and $\mathbf{T}$ but contains no Ric $*$ Ric term.

On the other hand

$$
\frac{\partial}{\partial t} \Gamma_{i j}^{k}=g^{k l}\left(h_{i l, j}+h_{j l, i}-h_{i j, l}\right),
$$

So the degree of $\left(\frac{\partial}{\partial t}-\Delta\right) \nabla \mathbf{T}$ is degree 4 but it contains no $\nabla^{2} \mathrm{Rm}$ or $\nabla^{3} \mathbf{T}$ term.

Therefore, all the terms $\left(\frac{\partial}{\partial t}-\Delta\right)|\mathrm{Rm}|^{2}+2|\nabla \mathrm{Rm}|^{2},\left(\frac{\partial}{\partial t}-\Delta\right)|\mathbf{T}|^{4}$ and $\left(\frac{\partial}{\partial t}-\Delta\right)|\nabla \mathbf{T}|^{2}+2\left|\nabla^{2} \mathbf{T}\right|^{2}$ can be bounded by

$$
\epsilon\left(|\nabla \mathrm{Rm}|^{2}+\left|\nabla^{2} \mathbf{T}\right|^{2}\right)+C_{\epsilon}\left(|\mathrm{Rm}|^{2}+|\mathbf{T}|^{4}+|\nabla \mathbf{T}|^{2}+1\right)^{3 / 2} .
$$


Choose $\epsilon=1$, then

$$
\begin{aligned}
&\left(\frac{\partial}{\partial t}-\Delta\right)\left(|\mathrm{Rm}|^{2}+|\mathbf{T}|^{4}+\right.\left.|\nabla \mathbf{T}|^{2}+1\right) \leq-\left(|\nabla \mathrm{Rm}|^{2}+\left|\nabla^{2} \mathbf{T}\right|^{2}\right) \\
&+C\left(|\mathrm{Rm}|^{2}+|\mathbf{T}|^{4}+|\nabla \mathbf{T}|^{2}+1\right)^{3 / 2}
\end{aligned}
$$

Similarly, for all $k=1,2,3 \ldots$, both the degree of $\left(\frac{\partial}{\partial t}-\Delta\right) \nabla^{k} \mathrm{Rm}$ and the degree of $\left(\frac{\partial}{\partial t}-\Delta\right) \nabla^{k+1} \mathbf{T}$ are $k+4$ but they contain no $\nabla^{k+2} \mathrm{Rm}$ or $\nabla^{k+3} \mathbf{T}$ term. So

$$
\begin{aligned}
& \left(\frac{\partial}{\partial t}-\Delta\right)\left(\left|\nabla^{k} \mathrm{Rm}\right|^{2}+\left|\nabla^{k+1} \mathbf{T}\right|^{2}\right) \leq-\left(\left|\nabla^{k+1} \mathrm{Rm}\right|^{2}+\left|\nabla^{k+2} \mathbf{T}\right|^{2}\right) \\
& \quad+C(k)\left(\sum_{j=0}^{k}\left(\left|\nabla^{j} \mathrm{Rm}\right|^{\frac{2(k+3)}{j+2}}+\left|\nabla^{j+1} \mathbf{T}\right|^{\frac{2(k+3)}{j+2}}\right)+|\mathbf{T}|^{2(k+3)}+1\right) .
\end{aligned}
$$

Let $Q=\left(\mu+|\mathrm{Rm}|^{2}+|\mathbf{T}|^{4}+|\nabla \mathbf{T}|^{2}\right)\left(|\nabla \mathrm{Rm}|^{2}+\left|\nabla^{2} \mathbf{T}\right|^{2}\right)$, then

$$
\begin{aligned}
\left(\frac{\partial}{\partial t}-\Delta\right) Q= & {\left[\left(\frac{\partial}{\partial t}-\Delta\right)\left(\mu+|\mathrm{Rm}|^{2}+|\mathbf{T}|^{4}+|\nabla \mathbf{T}|^{2}\right)\right]\left(|\nabla \mathrm{Rm}|^{2}+\left|\nabla^{2} \mathbf{T}\right|^{2}\right) } \\
& +\left(\mu+|\mathrm{Rm}|^{2}+|\mathbf{T}|^{4}+|\nabla \mathbf{T}|^{2}\right)\left(\frac{\partial}{\partial t}-\Delta\right)\left(|\nabla \mathrm{Rm}|^{2}+\left|\nabla^{2} \mathbf{T}\right|^{2}\right) \\
& -\left[\nabla\left(\mu+|\mathrm{Rm}|^{2}+|\mathbf{T}|^{4}+|\nabla \mathbf{T}|^{2}\right)\right]\left[\nabla\left(|\nabla \mathrm{Rm}|^{2}+\left|\nabla^{2} \mathbf{T}\right|^{2}\right)\right] \\
\leq & -\left(|\nabla \mathrm{Rm}|^{2}+\left|\nabla^{2} \mathbf{T}\right|^{2}\right)^{2}-\mu\left(\left|\nabla^{2} \mathrm{Rm}\right|^{2}+\left|\nabla^{3} \mathbf{T}\right|^{2}\right) \\
& +C(\Lambda, \mu)\left(|\nabla \mathrm{Rm}|^{2}+\left|\nabla^{2} \mathbf{T}\right|^{2}\right)^{\frac{4}{3}}+C(\Lambda, \mu) \\
& +C(\Lambda)\left(|\nabla \mathrm{Rm}|+\left|\nabla^{2} \mathbf{T}\right|+1\right)^{2}\left(\left|\nabla^{2} \mathrm{Rm}\right|+\left|\nabla^{3} \mathbf{T}\right|\right) .
\end{aligned}
$$

Choose $\mu=C(\Lambda)$ large enough so that

$$
\begin{aligned}
C(\Lambda)\left(|\nabla \mathrm{Rm}|+\left|\nabla^{2} \mathbf{T}\right|+1\right)^{2}\left(\left|\nabla^{2} \mathrm{Rm}\right|+\left|\nabla^{3} \mathbf{T}\right|\right) \leq & \frac{1}{4}\left(|\nabla \mathrm{Rm}|^{2}+\left|\nabla^{2} \mathbf{T}\right|^{2}\right)^{2} \\
& +\mu\left(\left|\nabla^{2} \mathrm{Rm}\right|^{2}+\left|\nabla^{3} \mathbf{T}\right|^{2}\right)^{\prime}
\end{aligned}
$$

then

$$
\begin{aligned}
\left(\frac{\partial}{\partial t}-\Delta\right) Q & \leq-\frac{3}{4}\left(|\nabla \mathrm{Rm}|^{2}+\left|\nabla^{2} \mathbf{T}\right|^{2}\right)^{2}+C(\Lambda)\left(|\nabla \mathrm{Rm}|^{2}+\left|\nabla^{2} \mathbf{T}\right|^{2}+1\right)^{\frac{4}{3}} \\
& \leq-\frac{1}{2}\left(|\nabla \mathrm{Rm}|^{2}+\left|\nabla^{2} \mathbf{T}\right|^{2}\right)^{2}+C(\Lambda) \\
& \leq-C(\Lambda) Q^{2}+C(\Lambda) \\
& =-C_{1}(\Lambda) Q^{2}+C_{2}(\Lambda) .
\end{aligned}
$$

Let $\phi$ be a cut-off function which is 0 outside $B_{r}$, and is 1 inside $B_{r / 2}$. We are done if we can find $\nu$ such that

$$
H=\frac{\nu}{\phi^{2}}+\frac{1}{C_{1}(\Lambda) t}+\sqrt{\frac{C_{2}(\Lambda)}{C_{1}(\Lambda)}}
$$


satisfies

$$
\left(\frac{\partial}{\partial t}-\Delta\right) H>-C_{1}(\Lambda) H^{2}+C_{2}(\Lambda)
$$

as long as $Q \leq H$

However

$$
\begin{gathered}
\frac{\partial}{\partial t} H=-\frac{1}{C_{1}(\Lambda) t^{2}}, \\
H^{2} \geq \frac{\nu^{2}}{\phi^{4}}+\frac{1}{C_{1}(\Lambda)^{2} t^{2}}+\frac{C_{2}(\Lambda)}{C_{1}(\Lambda)},
\end{gathered}
$$

and

$$
\begin{aligned}
\Delta H & =\nu \Delta \frac{1}{\phi^{2}} \\
& =\nu \nabla\left(-2 \frac{\nabla \phi}{\phi^{3}}\right) \\
& =\frac{\nu}{\phi^{4}}\left(-2 \phi \Delta \phi+6|\nabla \phi|^{2}\right) .
\end{aligned}
$$

So if $C_{1}(\Lambda) \nu>-2 \phi \Delta \phi+6|\nabla \phi|^{2}$ as long as $Q \leq H$, we are done.

Let $\tilde{g}$ be the metric at time 0 , let $\gamma$ be the distance to $p$ with respect to $\tilde{g}$. Pick a non-increasing cut-off function $\eta$ which is 0 on $\left[r^{2}, \infty\right)$ and is 1 on $\left[0, r^{2} / 4\right]$. Let $\phi=\eta\left(\gamma^{2}\right)$. Then for the ordinary derivatives

$$
\begin{gathered}
\partial_{i} \phi=2 \eta^{\prime}\left(\gamma^{2}\right) \gamma \partial_{i} \gamma \\
\partial_{i} \partial_{j} \phi=2 \eta^{\prime}\left(\gamma^{2}\right) \gamma \partial_{i} \partial_{j} \gamma+\left(4 \eta^{\prime \prime}\left(\gamma^{2}\right) \gamma^{2}+2 \eta^{\prime}\left(\gamma^{2}\right)\right) \partial_{i} \gamma \partial_{j} \gamma
\end{gathered}
$$

By Hessian comparison theorem,

$$
\tilde{\nabla}_{i j}^{2} \gamma=\partial_{i} \partial_{j} \gamma-\tilde{\Gamma}_{i j}^{p} \partial_{p} \gamma \leq C(\Lambda) \tilde{g}_{i j} / \gamma
$$

So

$$
\Delta \gamma=g^{i j}\left(\partial_{i} \partial_{j} \gamma-\tilde{\Gamma}_{i j}^{p} \partial_{p} \gamma\right) \leq C(\Lambda) g^{i j} \tilde{g}_{i j} / \gamma+g^{i j}\left(\tilde{\Gamma}_{i j}^{p}-\Gamma_{i j}^{p}\right) \partial_{p} \gamma .
$$

Since $\left|\frac{\partial}{\partial t} g_{i j}\right| \leq C(\Lambda)$, we see that $C(\Lambda, T)^{-1} \tilde{g}_{i j} \leq g_{i j} \leq C(\Lambda, T) \tilde{g}_{i j}$.

On the other hand the degree of

$$
\frac{\partial}{\partial t} \Gamma_{i j}^{k}=g^{k l}\left(h_{i l, j}+h_{j l, i}-h_{i j, l}\right)
$$

is 3, so it is bounded by $C(\Lambda, T)\left(|\nabla \mathrm{Rm}|+\left|\nabla^{2} \mathbf{T}\right|+1\right)$. Using $Q \leq H$, we see that

$$
\left|\frac{\partial}{\partial t} \Gamma_{i j}^{k}\right| \leq C(\Lambda, T)\left(\frac{\sqrt{\nu}}{\phi}+\frac{1}{\sqrt{t}}+1\right) .
$$

So

$$
\Delta \gamma \leq \frac{C(\Lambda, T)}{\gamma}+C(\Lambda, T)\left(\frac{\sqrt{\nu}}{\phi}+1\right)
$$

and

$$
\Delta \phi \geq-C(\Lambda, T, r)\left(\frac{\sqrt{\nu}}{\phi}+1\right)
$$


Therefore

$$
-2 \phi \Delta \phi+6|\nabla \phi|^{2} \leq C(\Lambda, T, r)(\sqrt{\nu}+1) .
$$

So if we choose $\nu=C(\Lambda, T, r)$ large enough, then

$$
C(\Lambda, T, r)(\sqrt{\nu}+1)<C_{1}(\Lambda) \nu
$$

can be achieved. We are done for the bound of $\left(|\nabla \mathrm{Rm}|^{2}+\left|\nabla^{2} \mathbf{T}\right|^{2}\right)$. Using

$$
Q_{k}=\left(\mu_{k}+\left|\nabla^{k} \mathrm{Rm}\right|^{2}+\left|\nabla^{k+1} \mathbf{T}\right|^{2}\right)\left(\left|\nabla^{k+1} \mathrm{Rm}\right|^{2}+\left|\nabla^{k+2} \mathbf{T}\right|^{2}\right),
$$

we can get higher derivative bounds.

\section{Perelman's $\mathcal{W}$ functional}

In [10], Perelman introduced the $\mathcal{W}$ functional

$$
\mathcal{W}(g, f, \tau)=\int_{M}\left[\tau\left(R+|\nabla f|^{2}\right)+f-n\right](4 \pi \tau)^{-n / 2} e^{-f} d g .
$$

By routine calculations [8], if $\delta g_{i j}=v_{i j}, \delta f=h, v=g^{i j} v_{i j}, \delta \tau=\sigma$, then

$$
\begin{aligned}
\delta \mathcal{W}= & \int_{M}\left[\left(\frac{v}{2}-h-\frac{n \sigma}{2 \tau}\right)\left(\tau\left(R+2 \Delta f-|\nabla f|^{2}\right)+f-n\right)\right. \\
& \left.+\sigma\left(R+|\nabla f|^{2}\right)+h-\tau\left(R_{i j}+f_{i j}\right) v^{i j}\right](4 \pi \tau)^{-n / 2} e^{-f} d g .
\end{aligned}
$$

For a general geometric flow

$$
\frac{\partial}{\partial t} g_{i j}=-2 R_{i j}+E_{i j}
$$

let $f(t, p)$ solve the backwards heat equation:

$$
\left\{\begin{array}{l}
\frac{\partial}{\partial t} f=-\Delta f-R+\frac{1}{2} g^{i j} E_{i j}+\frac{n}{2 \tau}+|\nabla f|^{2} \\
\tau=T-t
\end{array}\right.
$$

where $T$ is any given real number.

Let $\varphi_{t}$ be the diffeomorphism generated by the time-dependent vector fields $-\nabla f$, define $\tilde{g}(t)=\varphi_{t}^{*} g(t)$, and $\tilde{f}(t)=\varphi_{t}^{*} f(t)$, then

$$
\left\{\begin{array}{l}
\frac{\partial}{\partial t} \tilde{g}_{i j}=-2 \tilde{R}_{i j}+\tilde{E}_{i j}-2 \tilde{f}_{i j}:=\tilde{v}_{i j} \\
\frac{\partial}{\partial t} \tilde{f}=-\tilde{\Delta} \tilde{f}-\tilde{R}+\frac{1}{2} \tilde{g}^{i j} \tilde{E}_{i j}+\frac{n}{2 \tau}:=\tilde{h},
\end{array}\right.
$$

where the quantities with $\sim$ sign are just the original quantities pulled back under $\varphi_{t}$. Since

$$
\mathcal{W}(g(t), f(t), \tau(t))=\mathcal{W}(\tilde{g}(t), \tilde{f}(t), \tau(t)),
$$


we could use the variation formula to obtain

$$
\begin{aligned}
& \frac{\mathrm{d}}{\mathrm{d} t} \mathcal{W}(g(t), f(t), \tau(t)) \\
& =\frac{\mathrm{d}}{\mathrm{d} t} \mathcal{W}(\tilde{g}(t), \tilde{f}(t), \tau(t)) \\
& =\int\left\{-\tau\left(\tilde{R}_{i j}+\tilde{f}_{i j}\right) \tilde{v}^{i j}+\sigma\left(\tilde{R}+|\tilde{\nabla} \tilde{f}|^{2}\right)+\tilde{h}\right. \\
& \left.+\left(\frac{\tilde{v}}{2}-\tilde{h}-\frac{n \sigma}{2 \tau}\right)\left(\tau\left(\tilde{R}+2 \tilde{\Delta} \tilde{f}-|\tilde{\nabla} \tilde{f}|^{2}\right)+\tilde{f}-n\right)\right\}(4 \pi \tau)^{-\frac{n}{2}} e^{-\tilde{f}} \mathrm{~d} \tilde{g} \\
& =\int\left\{2 \tau\left(\tilde{R}_{i j}+\tilde{f}_{i j}\right)\left(\tilde{R}^{i j}+\tilde{f}^{i j}-\frac{1}{2} \tilde{E}^{i j}\right)\right. \\
& \left.-\left(\tilde{R}+|\tilde{\nabla} \tilde{f}|^{2}\right)-\tilde{\Delta} \tilde{f}-\tilde{R}+\frac{1}{2} \tilde{g}^{i j} \tilde{E}_{i j}+\frac{n}{2 \tau}\right\}(4 \pi \tau)^{-\frac{n}{2}} e^{-\tilde{f}} \mathrm{~d} \tilde{g} \\
& =\int\left\{2 \tau\left|\tilde{R}_{i j}+\tilde{f}_{i j}\right|^{2}-2(\tilde{R}+\tilde{\Delta} \tilde{f})+\frac{n}{2 \tau}\right. \\
& \left.-\tau\left(\tilde{R}_{i j}+\tilde{f}_{i j}-\frac{\tilde{g}_{i j}}{2 \tau}\right) \tilde{E}^{i j}\right\}(4 \pi \tau)^{-\frac{n}{2}} e^{-\tilde{f}} \mathrm{~d} \tilde{g} \\
& =\int\left\{2 \tau\left|\tilde{R}_{i j}+\tilde{f}_{i j}-\frac{\tilde{g}_{i j}}{2 \tau}\right|^{2}-\tau\left(\tilde{R}_{i j}+\tilde{f}_{i j}-\frac{\tilde{g}_{i j}}{2 \tau}\right) \tilde{E}^{i j}\right\}(4 \pi \tau)^{-\frac{n}{2}} e^{-\tilde{f}} \mathrm{~d} \tilde{g} \\
& =\int\left\{2 \tau\left|\tilde{R}_{i j}+\tilde{f}_{i j}-\frac{\tilde{g}_{i j}}{2 \tau}-\frac{\tilde{E}_{i j}}{4}\right|^{2}-\frac{\tau}{8}|\tilde{E}|^{2}\right\}(4 \pi \tau)^{-\frac{n}{2}} e^{-\tilde{f}} \mathrm{~d} \tilde{g} \\
& =\int\left\{2 \tau\left|R_{i j}+f_{i j}-\frac{g_{i j}}{2 \tau}-\frac{E_{i j}}{4}\right|^{2}-\frac{\tau}{8}|E|^{2}\right\}(4 \pi \tau)^{-\frac{n}{2}} e^{-f} \mathrm{~d} g \\
& \geq-\frac{\tau}{8}\left(\sup _{M}|E|\right)^{2} \int_{M}(4 \pi \tau)^{-n / 2} e^{-f} \mathrm{~d} g \text {. }
\end{aligned}
$$

Now we are interested in the infimum

$$
\mu(g, \tau)=\inf _{\int(4 \pi \tau)^{-n / 2} e^{-f} \mathrm{~d} g=1} \mathcal{W}(g, f, \tau)
$$

Suppose $\tau_{1}<\tau_{2}$ and $f$ achieves the infimum at $T-\tau_{1}$. Then by solving

$$
\frac{\partial}{\partial t} f=-\Delta f-R+\frac{1}{2} g^{i j} E_{i j}+\frac{n}{2 \tau}+|\nabla f|^{2}
$$

backwards,

$$
\int(4 \pi \tau)^{-n / 2} e^{-f} \mathrm{~d} g=1
$$

is still true for all $\tau \in\left[\tau_{1}, \tau_{2}\right]$. So

$$
\mu\left(g\left(T-\tau_{2}\right), \tau_{2}\right) \leq \mu\left(g\left(T-\tau_{1}\right), \tau_{1}\right)+\frac{1}{8} \int_{\tau_{1}}^{\tau_{2}} \tau \sup _{t=T-\tau}|E|^{2} \mathrm{~d} \tau .
$$

\section{$4 \kappa$-non-collapsing theorem}

The original $\kappa$-non-collapsing theorem of Perelman for Ricci flow in [10] requires the Riemannian curvature bound. However, the definition can be modified to the following version: 
Definition 4.1. The Riemannian metric $g$ on $M^{n}$ is said to be $\kappa$-noncollapsing relative to upper bound of scalar curvature on the scale $\rho$ if for any $B_{g}(p, r) \subset M$ with $r<\rho$ such that $\sup _{B_{g}(p, r)} R_{g} \leq r^{-2}$, we have $\mathrm{Vol}_{g} B_{g}(p, r) \geq \kappa r^{n}$.

The $\kappa$-non-collapsing theorem relative to upper bound of scalar curvature for Ricci flow was proved by Perelman (Section 13 of [8]). The proof can be modified to get the following theorem using the quasi-monotonicity formula (61) in the previous section:

Theorem 4.2. Let $\frac{\partial}{\partial t} g_{i j}=-2 R_{i j}+E_{i j}$ be a geometric flow on a compact manifold $M^{n}$. Then there exists a positive function $\kappa$ with 4 variables such that if $0<\rho \leq \rho_{0}<\infty, 0<\frac{T}{2} \leq t_{0} \leq T<\infty$ and

$$
\int_{0}^{t_{0}}\left(t_{0}+\rho^{2}-t\right) \sup _{M}|E|^{2} \mathrm{~d} t<\infty
$$

then $g\left(t_{0}\right)$ is $\kappa\left(g(0), T, \rho_{0}, \int_{0}^{t_{0}}\left(t_{0}+\rho^{2}-t\right) \sup _{M}|E|^{2} \mathrm{~d} t\right)$-non-collapsing relative to upper bound of scalar curvature on scale $\rho$.

Proof. Fix a cut-off function $\chi(s)$ such that $\chi(s)=1$ when $|s| \leq \frac{1}{2}$, and $\chi(s)=0$ when $|s| \geq 1$. For any $g\left(t_{0}\right)$-metric ball $B(p, r)$ of radius $r<\rho$ which satisfies $R(x) \leq r^{-2}$ for every $x \in B(p, r)$, we can define

$$
u(x)=e^{L / 2} \chi\left(\frac{d(x, p)}{r}\right),
$$

where $L$ is chosen so that

$$
\left(4 \pi r^{2}\right)^{-n / 2} \int_{M} u^{2}=1
$$

In particular,

$$
\begin{gathered}
\operatorname{Vol}(B(p, r)) \geq e^{-L}(4 \pi)^{n / 2} r^{n}, \\
\operatorname{Vol}\left(B\left(p, \frac{r}{2}\right)\right) \leq e^{-L}(4 \pi)^{n / 2} r^{n} .
\end{gathered}
$$

By monotonicity of $\mu$,

$$
\begin{aligned}
\mathcal{W}\left(g\left(t_{0}\right), u, r^{2}\right) & \geq \mu\left(g\left(t_{0}\right), r^{2}\right) \\
& \geq \mu\left(g(0), t_{0}+r^{2}\right)-\frac{1}{8} \int_{t_{0}+r^{2}}^{r^{2}} \tau \sup _{t=t_{0}+r^{2}-\tau}|E|^{2} \mathrm{~d} \tau \\
& =\mu\left(g(0), t_{0}+r^{2}\right)-\frac{1}{8} \int_{0}^{t_{0}}\left(t_{0}+r^{2}-t\right)\left(\sup _{M}|E|^{2}\right) \mathrm{d} t \\
& \geq \mu_{0}-\frac{1}{8} \int_{0}^{t_{0}}\left(t_{0}+\rho^{2}-t\right)\left(\sup _{M}|E|^{2}\right) \mathrm{d} t \\
& =\mu_{1},
\end{aligned}
$$

where $\mu_{0}$ is the lower bound of $\mu(g(0), \tau)$ when $\tau \in\left[\frac{T}{2}, T+\rho_{0}^{2}\right]$. So

$$
\mu_{1} \leq \int_{M}\left(4 \pi r^{2}\right)^{-n / 2}\left[r^{2}\left(R u^{2}+4|\nabla u|^{2}\right)+u^{2}(-2 \ln u-n)\right] .
$$


$R<C r^{-2}$ in $B(p, r),-2 u^{2} \ln u=-2 u^{2}(L / 2+\ln \chi)$ and

$$
|\nabla u| \leq \frac{e^{L / 2}}{r}\left|\chi^{\prime}\left(\frac{d(x, p)}{r}\right)\right| \leq \frac{C e^{L / 2}}{r},
$$

So

$$
\mu_{1} \leq C-L+\frac{C e^{L} \operatorname{Vol} B(p, r)}{r^{n}} \leq C-L+\frac{C \operatorname{Vol}(B(p, r))}{\operatorname{Vol}\left(B\left(p, \frac{r}{2}\right)\right)} .
$$

Thus if $\frac{\operatorname{Vol}(B(p, r))}{\operatorname{Vol}\left(B\left(p, \frac{r}{2}\right)\right)}<3^{n}$, then $-L \geq C$, so $\operatorname{Vol}(B(p, r)) \geq C_{1} r^{n}$. Let $\kappa=\min \left(C_{1}, \frac{\omega}{2}\right)$, then we claim that $\operatorname{Vol}(B(p, r)) \geq \kappa r^{n}$. Otherwise, $\operatorname{Vol}(B(p, r))<\kappa r^{n}$, so $\frac{\operatorname{Vol}(B(p, r))}{\operatorname{Vol}\left(B\left(p, \frac{r}{2}\right)\right)} \geq 3^{n}$, so

$$
\operatorname{Vol}\left(B\left(p, \frac{r}{2}\right)\right) \leq 3^{-n} \kappa r^{n} \leq \kappa\left(\frac{r}{2}\right)^{n}
$$

We can apply the same thing for $\frac{r}{2^{k}}$ and obtain that

$$
\operatorname{Vol}\left(B\left(p, \frac{r}{2^{k}}\right)\right) \leq \kappa\left(\frac{r}{2^{k}}\right)^{n},
$$

which is a contradiction.

\section{$5 \quad$ Finite time singularity}

Now we are ready to study the finite time singularities of reasonable flows of $\mathrm{G}_{2}$ structures.

First of all, using the method of Lotay-Wei and our Shi-type estimate, we can prove the following theorem:

Theorem 5.1. If $\phi(t)$ is a solution to a reasonable flow of $G_{2}$ structures on a compact manifold $M^{7}$ in a finite maximal time interval $[0, T)$, then

$$
\sup _{M}\left(|\mathrm{Rm}|^{2}+|\mathbf{T}|^{4}+|\nabla \mathbf{T}|^{2}\right)^{\frac{1}{2}} \geq \frac{C}{T-t}
$$

for some constant $C>0$.

Proof. As Lotay-Wei did in $\left[9\right.$, if $\sup _{M}\left(|\mathrm{Rm}|^{2}+|\mathbf{T}|^{4}+|\nabla \mathbf{T}|^{2}\right)^{\frac{1}{2}}$ is bounded, then all the higher order derivatives are also bounded. So $\frac{\partial}{\partial t} g$ and $\frac{\partial}{\partial t} \phi$ are all bounded. So they and their higher order derivatives are all bounded using the background metric $g(0)$. So we can take the smooth limit. This will violate the short-time existence assumption.

Still as Lotay-Wei, we can use the equation (30) to get the required blow-up rate.

Then we can get the following estimate:

Theorem 5.2. If $\phi(t)$ is a solution to a reasonable flow of $G_{2}$ structures on a compact manifold $M^{7}$ in a finite maximal time interval $[0, T)$, then

$$
\int_{0}^{T} \sup _{M}\left(|\mathrm{Ric}|+|\mathbf{T}|^{2}\right) \mathrm{d} t=\infty .
$$


Proof. If it is not true, then using the evolution equation, we can see that $\frac{\partial}{\partial t} \phi$ is bounded. So the metric is uniformly continuous. Using the proof of Theorem 8.1 of [9] as well as the Shi-type estimate, we can get a contradiction.

As for the better estimates of Ricci curvature, scalar curvature and torsion tensor, we can prove the following theorem using the method in 12

Theorem 5.3. Let $\phi(t)$ be a solution to a reasonable flow of $G_{2}$ structures on a compact manifold $M^{7}$ in a finite maximal time interval $[0, T)$. Assume that

$$
\int_{0}^{T}(T-t) \sup _{M}|\mathbf{T}|^{4} \mathrm{~d} t<\infty
$$

then

$$
\limsup _{t \rightarrow T}(T-t) \sup _{M}\left(|\operatorname{Ric}|+|\mathbf{T}|^{2}\right)>0
$$

and

$$
\limsup _{t_{0} \rightarrow T}\left[\left(T-t_{0}\right)^{2} \sup _{t \leq t_{0}}\left(1+|R|+|\mathbf{T}|^{2}\right) \sup _{t \leq t_{0}}\left(|\mathrm{Rm}|+|\mathbf{T}|^{2}+|\nabla \mathbf{T}|\right)\right]>0 .
$$

Proof. In this case, the flow is $\kappa$-non-collapsing on the scale $\sqrt{T-t}$. Using Shi-type estimate and the method of Wang in 12, it is easy to see that $\lim \sup _{t \rightarrow T}(T-t) \sup _{M}\left|\frac{\partial}{\partial t} g\right|>0$. So the first estimate is immediate.

As for the second estimate, we need to show that when $T-t_{0}<1$,

$$
\sup _{t \leq t_{0}}\left(|\operatorname{Ric}|+|\mathbf{T}|^{2}\right) \leq C \sqrt{O\left(t_{0}\right) Q\left(t_{0}\right)}
$$

where

$$
Q\left(t_{0}\right)=\sup _{t \leq t_{0}}\left(|\mathrm{Rm}|+|\mathbf{T}|^{2}+|\nabla \mathbf{T}|\right),
$$

and

$$
O\left(t_{0}\right)=\sup _{t \leq t_{0}}\left(1+|R|+|\mathbf{T}|^{2}\right) \leq 1+100 Q\left(t_{0}\right) .
$$

We will still follow the method in 12 . By Theorem 5.1 we see that $Q\left(t_{0}\right) \geq C(T-t)^{-1}$. So the flow is $\kappa$-non-collapsing on the scale $Q\left(t_{0}\right)^{-\frac{1}{2}}$. Now we re-scale the flow so that $Q\left(t_{0}\right)=1$. Then the harmonic radius has a lower bound. So inside a finite size of ball, the metric and all of its higher derivatives are uniformly bounded. So $\left(\frac{\partial}{\partial t}-D\right) \mathbf{T}=0$ for some elliptic operator $D$ with bounded coefficients and higher derivatives of coefficients. So after re-scaling,

$$
\sup \left|\nabla^{k} \mathbf{T}\right| \leq C(k) \sup |\mathbf{T}|
$$

Now

$$
\begin{aligned}
\frac{\partial}{\partial t} R= & -2 \Delta \operatorname{Tr} h+2 \operatorname{div}(\operatorname{div} h)-2<h, \operatorname{Ric}> \\
= & 2 \Delta R-2 \operatorname{div}(\operatorname{div} \operatorname{Ric})+2|\operatorname{Ric}|^{2}+\frac{L(\operatorname{Ric})}{Q\left(t_{0}\right)}+\frac{\mathbf{T} * \operatorname{Ric}}{\sqrt{Q\left(t_{0}\right)}}+\mathbf{T} * \mathbf{T} * \operatorname{Ric} \\
& +L\left(\nabla^{2}\left(\frac{C}{Q\left(t_{0}\right)}+\frac{\mathbf{T}}{\sqrt{Q\left(t_{0}\right)}}+\mathbf{T} * \mathbf{T}\right)\right) .
\end{aligned}
$$


The terms $2 \Delta R-2 \operatorname{div}(\operatorname{divRic})+2|\mathrm{Ric}|^{2}$ are equal to $\Delta R+2|\mathrm{Ric}|^{2}$ by Bianchi identity. The terms $L(\mathrm{Ric})+\mathbf{T} * \mathrm{Ric}+\mathbf{T} * \mathbf{T} * \mathrm{Ric}$ are bounded by $\frac{C O\left(t_{0}\right)}{Q\left(t_{0}\right)}|\mathrm{Ric}| \leq|\mathrm{Ric}|^{2}+C \frac{O\left(t_{0}\right)}{Q\left(t_{0}\right)}$. The rest terms are bounded by $C \frac{O\left(t_{0}\right)}{Q\left(t_{0}\right)}$.

For any $p$, we can pick a cut-off function $\chi$ such that it is 0 outside

$$
B_{g\left(t_{0}\right)}\left(p, Q\left(t_{0}\right)^{-1 / 2}\right) \times\left[t_{0}-\frac{1}{Q\left(t_{0}\right)}, t_{0}\right],
$$

and is 1 inside

$$
B_{g\left(t_{0}\right)}\left(p, \frac{1}{2} Q\left(t_{0}\right)^{-1 / 2}\right) \times\left[t_{0}-\frac{1}{2 Q\left(t_{0}\right)}, t_{0}\right] .
$$

After re-scaling, it vanishes outside $B_{g(0)}(p, 1) \times[-1,0]$ and is 1 inside $B_{g(0)}\left(p, \frac{1}{2}\right) \times\left[-\frac{1}{2}, 0\right]$.

Thus

$$
\begin{aligned}
\int_{t=0} \chi R & =\int_{-1}^{0}\left(\int_{M} \frac{\partial}{\partial t}(\chi R)\right) \mathrm{d} t \\
& =\int_{-1}^{0} \int_{M}\left[R\left(\frac{\partial}{\partial t}-\Delta\right) \chi+\chi\left(\frac{\partial}{\partial t}-\Delta\right) R\right] \mathrm{d} t .
\end{aligned}
$$

Since the geometry is bounded, it is easy to see that

$$
\int_{B_{g(0)}\left(p, \frac{1}{2}\right) \times\left[-\frac{1}{2}, 0\right]}|\mathrm{Ric}|^{2} \leq C \frac{O\left(t_{0}\right)}{Q\left(t_{0}\right)} .
$$

Now the Ricci curvature satisfies the equation

$$
\left|\left(\frac{\partial}{\partial t}-D\right) \operatorname{Ric}\right| \leq C \frac{O\left(t_{0}\right)}{Q\left(t_{0}\right)}
$$

for some elliptic operator $D$ with bounded coefficients and higher derivatives of coefficients. Therefore, we have $\mid$ Ric $\left.\right|^{2} \leq C \frac{O\left(t_{0}\right)}{Q\left(t_{0}\right)}$. Before re-scaling, it is exactly $|\mathrm{Ric}| \leq C \sqrt{O\left(t_{0}\right) Q\left(t_{0}\right)}$.

If in addition

$$
\sup _{M}\left(|R|+|\mathbf{T}|^{2}\right)=o\left(\frac{1}{T-t}\right),
$$

we can also show that any blow-up limit at finite time must be a manifold with maximal volume growth rate whose holonomy is contained in $\mathrm{G}_{2}$.

Theorem 5.4. Let $\phi(t)$ be a solution to a reasonable flow of $G_{2}$ structures on a compact manifold $M^{7}$ in a finite maximal time interval $[0, T)$. If

$$
\int_{0}^{T}(T-t) \sup _{M}|\mathbf{T}|^{4} \mathrm{~d} t<\infty,
$$

and

$$
\sup _{M}\left(|R|+|\mathbf{T}|^{2}\right)=o\left(\frac{1}{T-t}\right),
$$

then there exists a sequence $t_{k} \rightarrow T, p_{k} \in M$ such that

$$
Q_{k}=\left(|\mathrm{Rm}|^{2}+|\mathbf{T}|^{4}+|\nabla \mathbf{T}|^{2}\right)^{\frac{1}{2}}\left(p_{k}, t_{k}\right) \rightarrow \infty,
$$


and $\left(M, Q_{k}^{3 / 2} \phi\left(t_{k}\right), Q_{k} g\left(t_{k}\right), p_{k}\right)$ converges to a complete manifold $M_{\infty}$ with a torsion-free $G_{2}$ structure $\left(\phi_{\infty}, g_{\infty}, p_{\infty}\right)$ such that

$$
\operatorname{Vol}_{g_{\infty}}\left(B_{g_{\infty}}\left(p_{\infty}, r\right)\right) \geq \kappa r^{7}
$$

for some $\kappa>0$ and all $r>0$.

Proof. First of all, we can see that

$$
\limsup _{t_{0} \rightarrow T}\left[\left(T-t_{0}\right) \sup _{t \leq t_{0}}\left(|\mathrm{Rm}|+|\mathbf{T}|^{2}+|\nabla \mathbf{T}|\right)\right]=\infty .
$$

So we can choose a sequence such that $\left(T-t_{k}\right) Q_{k} \rightarrow \infty$. After re-scaling, $\left(|\mathrm{Rm}|^{2}+|\mathbf{T}|^{4}+|\nabla \mathbf{T}|^{2}\right)^{\frac{1}{2}}$ is bounded. Moreover, $\sup \left(|R|+|\mathbf{T}|^{2}\right)$ converges to 0 , and the manifold is $\kappa$-non-collapsing on a scale going to infinity. In particular, we get a uniform volume lower bound in any finite scale. Therefore, by our Shi-type estimate, the $\mathrm{G}_{2}$ structures converge in $C^{\infty}$ sense to a limit $\mathrm{G}_{2}$ structure. In the limit, both the scalar curvature and the torsion tensor are everywhere 0 . In other words, the limit is torsionfree. Moreover, it has maximal volume growth rate.

Acknowledgement: The author is grateful to the helpful discussions with Xiuxiong Chen, Jason Lotay and Chengjian Yao.

\section{References}

[1] Bryant, Robert L.: Some remarks on $\mathrm{G}_{2}$-structures. Proceedings of Gökova Geometry-Topology Conference 2005, 75-109, Gökova Geometry/Topology Conference (GGT), Gökova, 2006.

[2] Bryant, Robert L.; Xu, Feng: Laplacian Flow for Closed $\mathrm{G}_{2^{-}}$ Structures: Short Time Behavior. Preprint, arXiv:1101.2004

[3] Chow, Bennett; Knopf, Dan: The Ricci flow: an introduction. Mathematical Surveys and Monographs, 110. American Mathematical Society, Providence, RI, 2004.

[4] Fernández, Marisa; Gray, Alfred: Riemannian manifolds with structure group $\mathrm{G}_{2}$. Ann. Mat. Pura Appl. (4) 132 (1982), 19-45 (1983).

[5] Grigorian, Sergey: Short-time behaviour of a modified Laplacian coflow of $\mathrm{G}_{2}$-structures. Adv. Math. 248 (2013), 378-415.

[6] Karigiannis, Spiro: Flows of $\mathrm{G}_{2}$-structures. I. Q. J. Math. 60 (2009), no.4, 487-522.

[7] Karigiannis, Spiro; McKay, Benjamin; Tsui, Mao-Pei: Soliton solutions for the Laplacian co-flow of some $\mathrm{G}_{2}$-structures with symmetry. Differential Geom. Appl. 30 (2012), no.4, 318-333.

[8] Kleiner, Bruce; Lott, John: Notes on Perelman's papers. Geom. Topol. 12 (2008), no.5, 2587-2855.

[9] Lotay, Jason D.; Wei, Yong: Laplacian flow for closed $\mathrm{G}_{2}$ structures: Shi-type estimates, uniqueness and compactness. Geom. Funct. Anal. 27 (2017), no. 1, 165-233. 
[10] Perelman, Grisha: The entropy formula for the Ricci flow and its geometric applications. Preprint. arXiv:math/0211159

[11] Shi, Wan-Xiong: Deforming the metric on complete Riemannian manifolds. J. Differential Geom. 30 (1989), no.1, 223-301.

[12] Wang, Bing: On the conditions to extend Ricci flow (II). Int. Math. Res. Not. IMRN 2012, no.14, 3192-3223. 